\title{
Exercise to prevent falls in older adults: an updated systematic review and meta-analysis
}

\author{
Catherine Sherrington, ${ }^{1}$ Zoe A Michaleff, ${ }^{1,2}$ Nicola Fairhall, ${ }^{1}$ Serene S Paul, ${ }^{1}$ \\ Anne Tiedemann, ${ }^{1}$ Julie Whitney, ${ }^{3}$ Robert G Cumming, ${ }^{4}$ Robert D Herbert, ${ }^{5}$ \\ Jacqueline C T Close, ${ }^{5,6}$ Stephen R Lord ${ }^{5}$
}

- Additional material is

published online only. To view please visit the journal online (http://dx.doi.org/10.1136/ bjsports-2016-096547).

${ }^{1}$ The George Institute for Global Health, Sydney Medical School, The University of Sydney, Sydney, New South Wales, Australia

${ }^{2}$ Arthritis Research UK Primary Care Centre, Research Institute for Primary Care and Health Sciences, Keele University, UK ${ }^{3}$ Clinical Age Research Unit, King's College Hospital, London, UK

${ }^{4}$ School of Public Health, Sydney Medical School, The University of Sydney, Sydney, New South Wales, Australia

${ }^{5}$ Neuroscience Research

Australia, University of New South Wales, Sydney, New South

Wales, Australia

${ }^{6}$ Prince of Wales Clinical School, University of New South Wales, Sydney, New South Wales, Australia

Correspondence to Professor Catherine Sherrington, The George Institute for Global Health, Sydney Medical School The University of Sydney, NSW 2000, Australia;

csherrington@georgeinstitute. org.au

Accepted 25 August 2016 Published Online First 4 October 2016

\section{ABSTRACT}

Objective Previous meta-analyses have found that exercise prevents falls in older people. This study aimed to test whether this effect is still present when new trials are added, and it explores whether characteristics of the trial design, sample or intervention are associated with greater fall prevention effects.

Design Update of a systematic review with random effects meta-analysis and meta-regression.

Data sources Cochrane Library, CINAHL, MEDLINE, EMBASE, PubMed, PEDro and SafetyLit were searched from January 2010 to January 2016.

Study eligibility criteria We included randomised controlled trials that compared fall rates in older people randomised to receive exercise as a single intervention with fall rates in those randomised to a control group. Results 99 comparisons from 88 trials with 19478 participants were available for meta-analysis. Overall, exercise reduced the rate of falls in community-dwelling older people by $21 \%$ (pooled rate ratio $0.79,95 \% \mathrm{Cl}$ 0.73 to $0.85, p<0.001,1^{2} 47 \%, 69$ comparisons) with greater effects seen from exercise programmes that challenged balance and involved more than 3 hours/ week of exercise. These variables explained $76 \%$ of the between-trial heterogeneity and in combination led to a $39 \%$ reduction in falls (incident rate ratio $0.61,95 \% \mathrm{Cl}$ 0.53 to $0.72, p<0.001$ ). Exercise also had a fall prevention effect in community-dwelling people with Parkinson's disease (pooled rate ratio $0.47,95 \% \mathrm{Cl}$ 0.30 to $0.73, p=0.001,\left.\right|^{2} 65 \%, 6$ comparisons) or cognitive impairment (pooled rate ratio $0.55,95 \% \mathrm{Cl}$ 0.37 to $0.83, p=0.004, I^{2} 21 \%, 3$ comparisons). There was no evidence of a fall prevention effect of exercise in residential care settings or among stroke survivors or people recently discharged from hospital.

Summary/conclusions Exercise as a single intervention can prevent falls in community-dwelling older people. Exercise programmes that challenge balance and are of a higher dose have larger effects. The impact of exercise as a single intervention in clinical groups and aged care facility residents requires further investigation, but promising results are evident for people with Parkinson's disease and cognitive impairment.

\section{INTRODUCTION}

Falls are a leading cause of morbidity and mortality in older adults. ${ }^{1} 2$ Approximately one in three community-dwelling people aged 65 years or older will fall at least once per year, ${ }^{3}$ and the risk of falling increases with age. ${ }^{2}$ Falls impose a significant social and economic burden for individuals, their families, community health services and the economy. As the proportion of older people is rising globally, the costs associated with falls will increase. ${ }^{4}$ The prevention of falls is therefore an urgent public health challenge. National health bodies and international guidelines are promoting the implementation of appropriately designed intervention programmes that are known to prevent falls in older people. ${ }^{5} 6$

There is strong evidence that appropriately designed intervention programmes can prevent falls in older people. ${ }^{7-9}$ A Cochrane systematic review ${ }^{7}$ established that exercise interventions reduce the rate of falls (number of falls per person) and risk of falling (proportion of people having one or more falls) in community-dwelling older people. Furthermore, exercise as a single intervention has a fall prevention effect similar to multifaceted interventions, ${ }^{7} 10$ suggesting implementation of exercise as a stand-alone intervention may be the optimal and potentially most cost-effective ${ }^{11}$ approach to fall prevention at a population level.

Trials of exercise for fall prevention are heterogeneous in risk of bias, populations involved and content of exercise programmes. Meta-regression enables investigation of between-trial variability, that is, whether certain trial-level factors are associated with greater effects of intervention programmes. $^{12}$ Our previous meta-analyses with meta-regression, which included 44 trials in $2008^{8}$ and 54 trials in $2011,{ }^{9}$ found greater fall prevention effects in trials where exercise programmes included balance training, were undertaken more frequently (ie, exceeded 2 hours a week over the study period) and did not include walking exercise.

Recent publication of additional trials necessitates an update of the previous systematic review, meta-analyses and meta-regression. This systematic review and meta-analysis aimed to: (1) determine the effects of exercise on fall rates in older people when compared with no exercise in randomised controlled trials (RCTs), and (2) explore whether characteristics of the trial design, sample or intervention are associated with greater fall prevention effects.

\section{METHODS}

This systematic review was conducted in accordance with the PRISMA guidelines and the checklist was completed. ${ }^{13}$

\section{Inclusion and exclusion criteria}

RCTs conducted among older people (mean age $\geq 65$ years) in which the primary intervention being 
evaluated was exercise and the outcome was the number of falls, rate of falls or number of fallers were considered for inclusion. Only trials that reported the number or rate of falls were combined in the meta-analyses. Trials were ineligible if non-exercise interventions were a major ( $>25 \%$ of time) component of the intervention being evaluated or if the control group received exercise, unless the control group's exercise appeared to be of insufficient intensity (ie, upper limb or stretching exercise only, or authors stated there was no challenge to balance), dose (ie, less than four supervised sessions) and progression (ie, authors stated exercises were not progressed in terms of intensity or challenge to balance) to have beneficial effects on balance or mobility.

\section{Search strategy}

This review is an update of our two previously published systematic reviews. ${ }^{8} 9$ Seven electronic databases were searched (Cochrane Library, CINAHL, MEDLINE, EMBASE, PubMed, PEDro and SafetyLit) from 1 January 2010 to 22 January 2016. The search strategy consisted of terms describing the population, outcome and study type as shown in online supplementary file S1. The reference lists of included studies, relevant protocol papers and systematic reviews were screened and forward citation tracking was conducted to identify studies missed by the database search.

\section{Review process}

To determine eligibility of identified trial reports, two of the following five contributors (ZAM, SSP, NF, CS, Claire Mok) independently screened titles and abstracts. Full texts were obtained where necessary. Differences of opinion between authors about study eligibility were resolved by discussion and adjudicated by the lead author (CS) as required.

\section{Risk of bias}

Risk of bias was assessed using the PEDro scale. ${ }^{14}$ When available, the PEDro rating and score was obtained from the PEDro database. ${ }^{15}$ In all other instances, two authors independently rated risk of bias. Differences were resolved by discussion and adjudicated by the lead author (CS) as required.

\section{Study analysis}

Two of the following five contributors (ZAM, SSP, NF, JW, Angie Baba) extracted data on study design, sample characteristics, intervention design and estimates of effect of exercise from each study. The studies were described in terms of trial design (sample size, follow-up period, PEDro score), sample characteristics (dwelling type, clinical condition, age, control group fall rate, falls risk status), intervention components (strength training, balance training, endurance training, flexibility exercise, walking training or practice, amount of supervision,

Table 1 Summary of included comparisons ( $n=99$ comparisons in 88 trials) grouped by residence and health condition

\begin{tabular}{|c|c|c|c|c|c|c|}
\hline Residence/health condition* & $\begin{array}{l}\text { Residential } \\
\text { care }\end{array}$ & $\begin{array}{l}\text { General } \\
\text { community }\end{array}$ & $\begin{array}{l}\text { Parkinson's } \\
\text { disease }\end{array}$ & Stroke & $\begin{array}{l}\text { Cognitive } \\
\text { impairment }\end{array}$ & $\begin{array}{l}\text { After hospital } \\
\text { discharge }\end{array}$ \\
\hline Number of comparisons & 15 & 69 & 6 & 3 & 3 & 3 \\
\hline Sample size at randomisation, mean (SD), total sample & $\begin{array}{l}134(147) \\
1876\end{array}$ & $\begin{array}{l}264(341) \\
15773\end{array}$ & $134(89) 669$ & $\begin{array}{l}126(35) \\
252\end{array}$ & $91(104) 272$ & $212(146) 636$ \\
\hline Follow-up (weeks), mean (SD) & $33(15)$ & $51(27)$ & $30(19)$ & $25(23)$ & $31(19)$ & $35(15)$ \\
\hline PEDro score, $\dagger$ mean (SD) & $6(2)$ & $6(1)$ & $7(1)$ & $8(0)$ & $7(1)$ & $8(1)$ \\
\hline Average age $>75$ years & 14 & 33 & 1 & 0 & 3 & 3 \\
\hline $\begin{array}{l}\text { Control group falls/person-year or proportion who fell } \\
\text { in follow-up period, mean (SD) }\end{array}$ & $1.8(1.4)$ & $1.0(0.9)$ & $16.1(13.7)$ & $2.1(0.3)$ & $1.5(1.6)$ & $2.2(1.3)$ \\
\hline $\begin{array}{l}\text { Unselected population (increased fall risk not an } \\
\text { inclusion criterion) }\end{array}$ & - & 37 & - & - & - & \\
\hline Moderate or high intensity strength training $\ddagger$ & 3 & 28 & 2 & 1 & 2 & 2 \\
\hline High intensity strength training§ & 1 & 10 & 1 & 0 & 0 & 1 \\
\hline Moderate or high challenge balance training & 9 & 47 & 5 & 2 & 3 & 2 \\
\hline High challenge balance training** & 5 & 31 & 3 & 1 & 3 & 2 \\
\hline Moderate or high intensity endurance training $\dagger \dagger$ & 2 & 14 & 1 & 0 & 0 & 0 \\
\hline Flexibility programme $\ddagger$ & 3 & 22 & 0 & 0 & 0 & 0 \\
\hline Walking programme§§ & 9 & 29 & 2 & 1 & 2 & 0 \\
\hline Ten exercise participants/instructorףף & 14 & 25 & 3 & 1 & 1 & 0 \\
\hline Exercises tailored in type or intensity*** & 8 & 31 & 5 & 1 & 3 & 3 \\
\hline $2+$ hours of exercise per weektt† & 5 & 40 & 6 & 3 & 3 & 2 \\
\hline $3+$ hours of exercise per week $\neq \ddagger \ddagger$ & 2 & 20 & 5 & 3 & 0 & 1 \\
\hline Good adherence§§§ & 13 & 52 & 6 & 3 & 3 & 1 \\
\hline
\end{tabular}

${ }^{*}$ Studies in the clinical populations were also among community dwellers.

tCoded using PEDro rating scale. ${ }^{14}$

$\ddagger$ §Moderate intensity ( $40-60 \%$ of the 1-repetition maximum (RM) that is, a weight so heavy that it can only be lifted once) or high intensity ( $>60 \% 1$ RM).

$\eta^{* *}{ }^{*}$ Moderately challenging=two of the following criteria or highly challenging=all three criteria: movement of the centre of mass, narrowing of the base of support and minimising upper limb support.

t+Moderate intensity $=40-60 \%$ maximum heart rate, some increase in breathing or heart rate, or perceived exertion of $11-14$ on the Borg scale or high intensity=above $60 \%$ of maximum heart rate or heart rate reserve, large increase in breathing or heart rate (conversation is difficult or broken) or perceived exertion of 15 or greater on the Borg scale.

$\ddagger \ddagger$ Short-duration or long-duration stretches specifically mentioned.

$\S \S$ Walking programme/practice was specifically mentioned.

ๆीTen or fewer participants per instructor.

$* * *$ Type or intensity of most exercises was designed for each individual based on an assessment.

$\dagger \dagger t, \ddagger \ddagger \ddagger G$ reater than or equal to 2 or 3 hours of exercise with instructor plus prescribed home exercise per week over intervention period.

$\S \S \S G$ reater than or equal to $75 \%$ participants attended $50 \%$ or more sessions and/or $>50 \%$ attendance rate. 
exercises tailored in type or intensity, dose of exercise) and adherence to programme. Some characteristics were coded on 3 - to 5-point scales, and were dichotomised for the analysis as shown in table 1.

Estimates of the effect of exercise on the rate of falls were extracted from each trial. Where possible, estimates of incident rate ratios (IRRs) from negative binomial regression models or HRs from proportional hazards models that allowed for multiple falls per person were used. Alternatively, data on the total number of falls per group or number of falls per person and exposure times (person-years of follow-up using actual follow-up times and number of participants providing data where reported) were used to calculate rate ratios. Where possible, unadjusted fall rates and longer follow-up times were used (eg, in an article which presented 6-month and 12-month fall data, the 12-month data were used) except where the long-term fall rates reported were more than 1 year after completion of the intervention. For trials with more than one exercise intervention group, separate estimates of the effects of each exercise intervention were calculated. To avoid 'double counting' of control participants from these trials, the total falls and participant numbers in the control group were allocated in proportion to the participant numbers in each intervention group. For trials that were cluster randomised but did not account for the effect of clustering, the variance of estimates was adjusted by assuming an intracluster correlation of 0.01 .

Random effects meta-analysis was undertaken using userwritten commands in Stata V.13 software (Stata Corp, College Station, Texas, USA). Pooled analyses were undertaken for (1) trials in general older community-dwelling populations and (2) trials conducted in residential care settings. Separate meta-analyses were undertaken for trials conducted in people with Parkinson's disease, stroke, cognitive impairment and people recently discharged from hospital. The pooled rate ratio was calculated and between-trial heterogeneity was determined by visual inspection of the forest plots and with consideration of the $\mathrm{I}^{2}$ statistic. The pooled effect was calculated using the 'metan' command. ${ }^{16}$ Egger's test and visual inspection of funnel plots were undertaken to assess small study effects. Sensitivity analyses were conducted to assess the effects of exercise on falls using fixed-effect meta-analysis and excluding in turn: smaller trials (samples sizes of $<200$ at randomisation); trials with a higher risk of bias (PEDro Scale score $<7$ ); trials for which the ratio comparing fall rates between groups was calculated by the review authors using the number of falls or rate of falls by group; and cluster randomised trials. Given the few studies in people with particular clinical conditions, sensitivity analyses for these meta-analyses were only undertaken excluding trials with a higher risk of bias (PEDro Scale score <7).

Meta-regression was undertaken using the user-written Stata command 'metareg' ${ }^{17}$ to explore the impact of trial-level characteristics relating to trial design (sample size, follow-up period, PEDro score), sample characteristics (average age, control group fall rate, use of unselected population rather than inclusion of individuals on the basis of an increased risk of falls), intervention components (moderate-intensity and/or high-intensity strength training, moderate-challenge and/or high-challenge balance training, walking training or practice, $2+$ or $3+$ hours of exercise intervention per week over the programme period) and better exercise adherence $(\geq 75 \%$ participants attended $50 \%$ or more sessions and/or $>50 \%$ attendance rate).

Figure 1 Flow diagram.

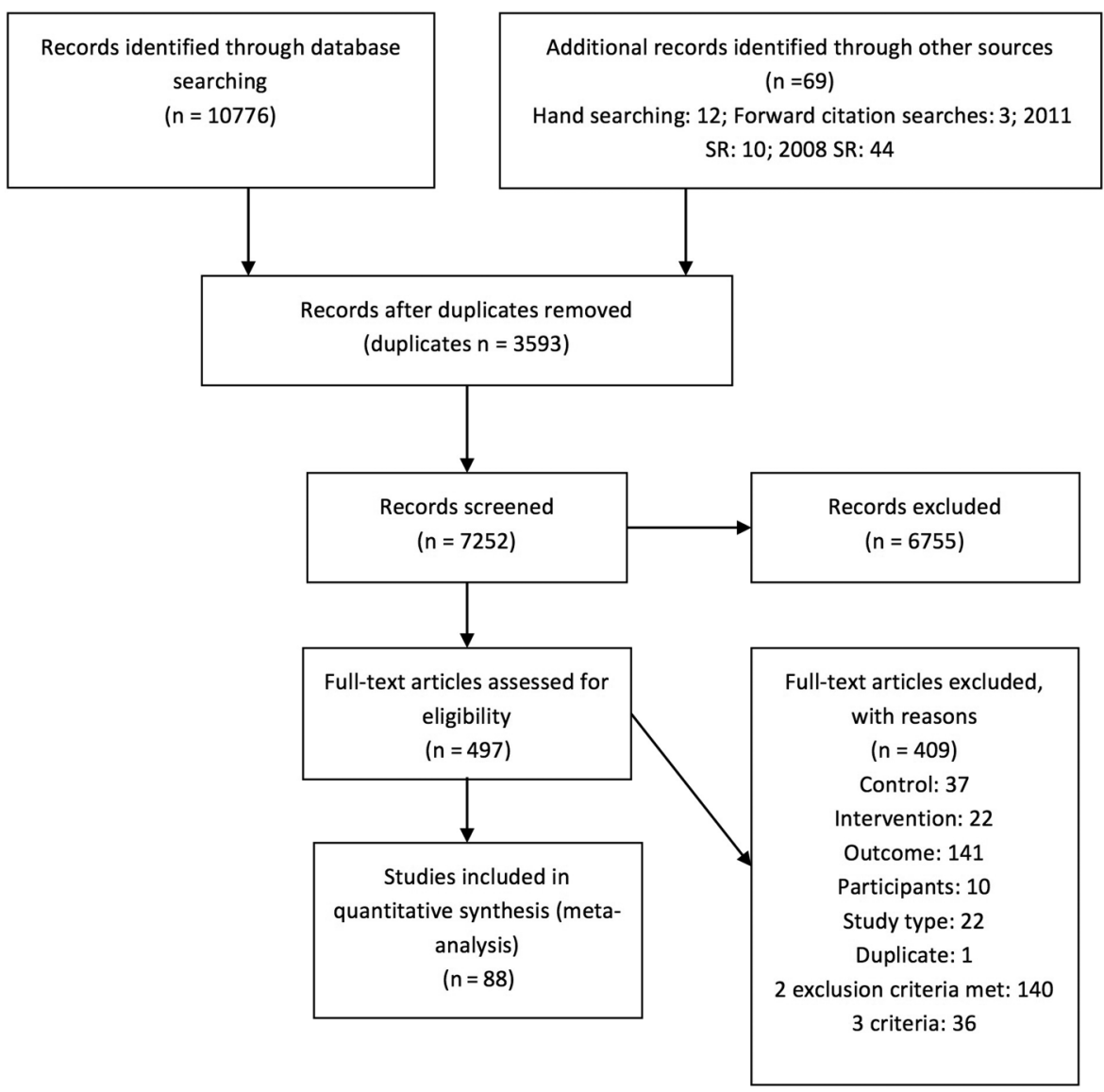




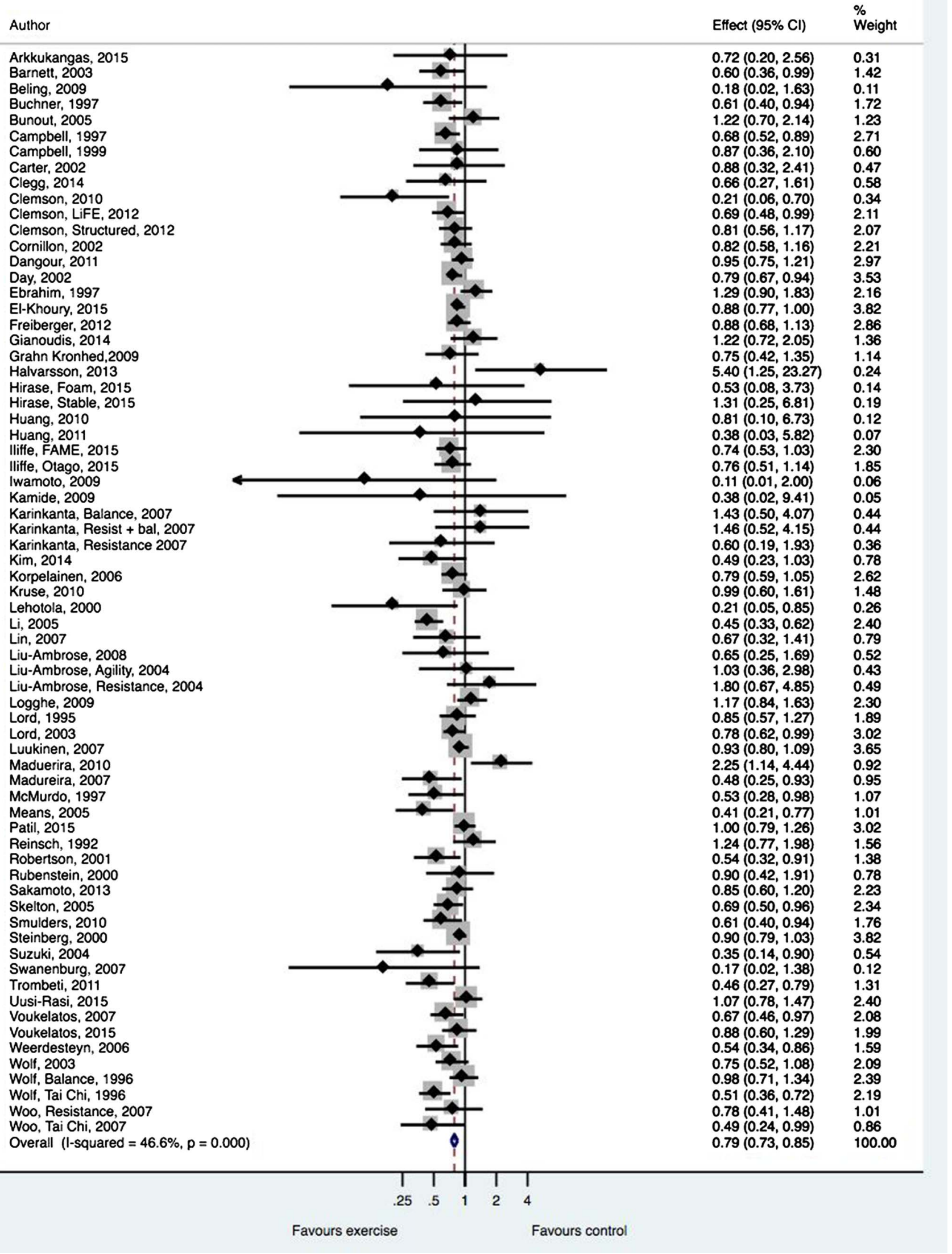

Figure 2 Forest plot of trials of exercise to prevent falls undertaken in general community dwellers. 


\section{RESULTS}

The electronic search retrieved 10776 articles (figure 1). After screening, 112 eligible RCTs were identified of which 88 trials provided data of the number of falls in each group so were included in the meta-analysis. Characteristics of the trials are shown in online supplementary file S2 and summarised in table 1.

Nine of the 88 trials included 2 exercise groups and 1 control group. One trial had 3 exercise groups and 1 control group, so 99 comparisons were available for meta-analysis. The included trials involved a total of 19478 participants. Most trials were conducted in people living in the general community (61 trials, 69 comparisons); 10 trials (10 comparisons) were conducted in high-care residential facilities (nursing homes) and 4 trials (5 comparisons) were conducted in low-care residential facilities (hostels). Two trials ${ }^{18}{ }^{19}$ were conducted predominantly in retirement villages or aged-care communities where older people cared for themselves, so these trials were included with general community analyses. Thirteen trials (15 comparisons)

Figure 3 Forest plot of trials of exercise to prevent falls undertaken in residential care settings.

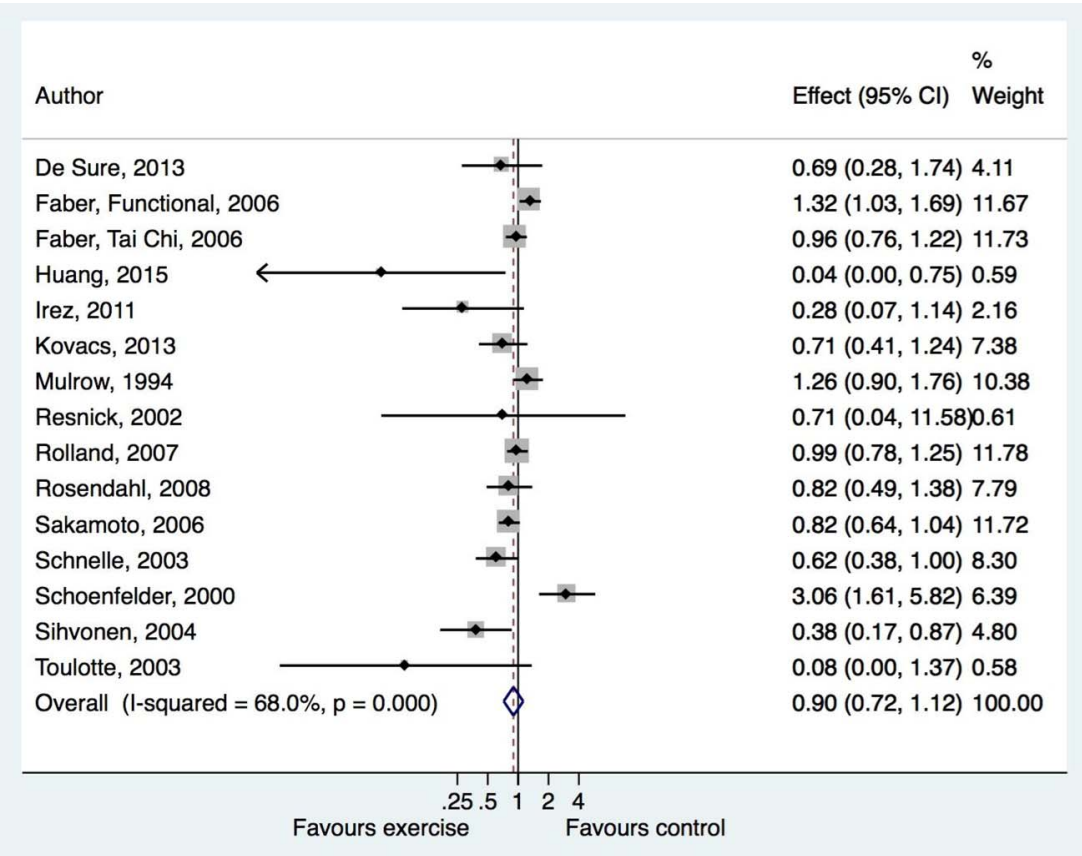

Table 2 Effect of exercise on falls, results of primary meta-analyses and sensitivity analyses

\begin{tabular}{|c|c|c|c|}
\hline Analysis & Number of comparisons & Pooled rate ratio, $95 \% \mathrm{Cl}$ & $I^{2}(\%)$ \\
\hline \multicolumn{4}{|l|}{ General community dwellers } \\
\hline Random effects meta-analysis all comparisons & 69 & $0.79(0.73$ to 0.85$)$ & 47 \\
\hline Fixed-effect meta-analysis all comparisons & 69 & $0.82(0.79$ to 0.86$)$ & 47 \\
\hline Random effects meta-analysis studies $n>200$ & 27 & 0.81 (0.75 to 0.89$)$ & 52 \\
\hline Random effects meta-analysis studies PEDro score $\geq 7^{*}$ & 24 & 0.81 (0.74 to 0.89 ) & 30 \\
\hline Random effects meta-analysis no computed analysest & 28 & $0.76(0.69$ to 0.83$)$ & 46 \\
\hline Random effects meta-analysis no cluster trials & 62 & $0.78(0.72$ to 0.85$)$ & 49 \\
\hline \multicolumn{4}{|l|}{ Residential care dwellers } \\
\hline Random effects meta-analysis all comparisons & 15 & $0.90(0.72$ to 1.12$)$ & 68 \\
\hline Fixed-effect meta-analysis all comparisons & 15 & 0.98 (0.88 to 1.08$)$ & 68 \\
\hline Random effects meta-analysis studies $n>200$ & 3 & 1.01 (0.77 to 1.33$)$ & 74 \\
\hline Random effects meta-analysis studies PEDro score $\geq 7^{*}$ & 4 & $0.82(0.57$ to 1.19$)$ & 48 \\
\hline Random effects meta-analysis no computed analyses & 1 & $0.82(0.49$ to 1.38$)$ & - \\
\hline Random effects meta-analysis no cluster trials & 14 & $0.92(0.77$ to 1.10$)$ & 65 \\
\hline \multicolumn{4}{|l|}{ Clinical populations (community dwellers) } \\
\hline Parkinson's disease, all trials & 6 & $0.47(0.30$ to 0.73$)$ & 65 \\
\hline Parkinson's disease, PEDro score $\geq 7^{*}$ & 4 & $0.44(0.23$ to 0.83$)$ & 79 \\
\hline Stroke, all trials & 3 & 0.74 (0.42 to 1.32$)$ & 39 \\
\hline Cognitive impairment, all trials & 3 & $0.55(0.37$ to 0.83$)$ & 21 \\
\hline Cognitive impairment, PEDro score $\geq 7^{*}$ & 2 & $0.50(0.40$ to 0.61$)$ & 0 \\
\hline After hospital discharge, all trialsł & 3 & $1.16(0.88$ to 1.52$)$ & 47 \\
\hline
\end{tabular}


A

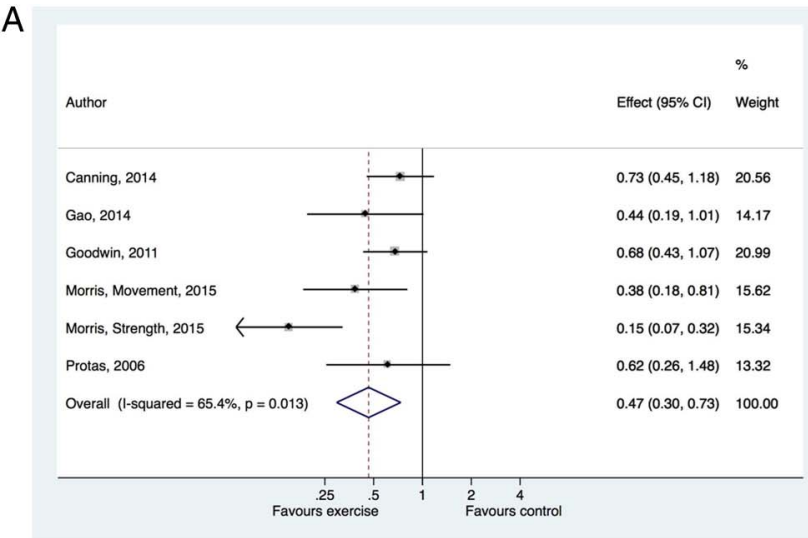

B

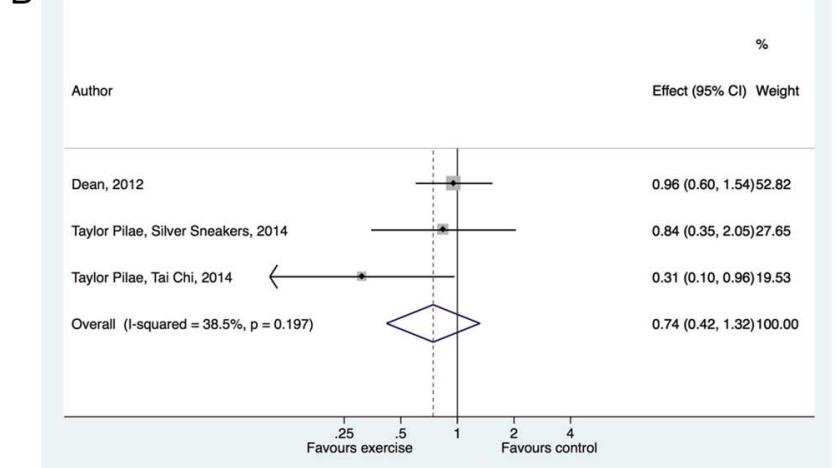

C
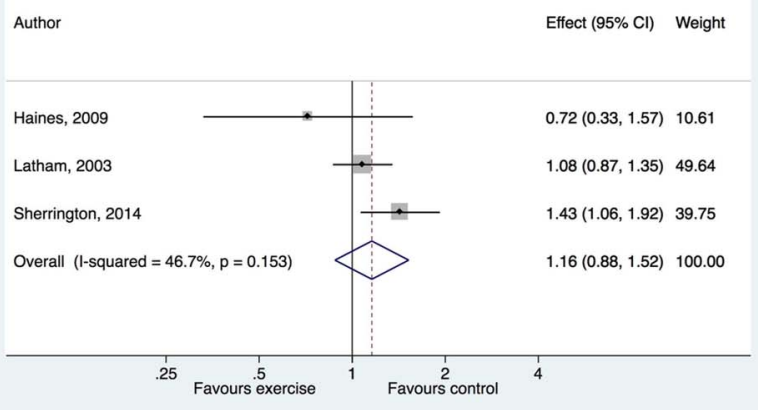

D

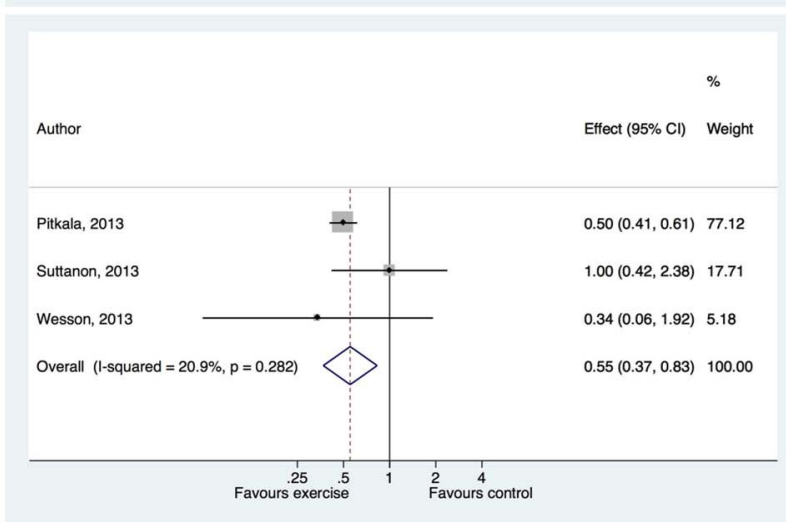

Figure 4 Forest plots of trials of exercise to prevent falls undertaken in community dwellers with clinical conditions. (A) Parkinson's disease, (B) stroke, (C) recent hospital discharge and (D) cognitive impairment.

included only community-dwelling participants with a clinical condition likely to increase the risk of falls (eg, Parkinson's disease, stroke, impaired cognition) or a specific recruitment method likely to indicate a high-risk population (recent discharge from hospital). Around half of the exercise programmes evaluated in the trials tailored the intensity or type of exercise to the individual $(n=51)$ and were conducted under supervision of an instructor, with $<10$ participants per instructor $(n=44)$.

\section{Effects of exercise on fall rates}

The pooled effect of exercise on fall rates in communitydwelling older people, expressed as a rate ratio, was $0.79(95 \%$ CI 0.73 to $0.85, \mathrm{p}<0.001, \mathrm{I}^{2} 47 \%, 69$ comparisons, figure 2) and in residential care settings was 0.90 (95\% CI 0.72 to 1.12, $\mathrm{p}=0.35, \mathrm{I}^{2} 68 \%, 15$ comparisons, figure 3$)$. The pooled estimate of the effect of exercise on falls in community-dwelling older people remained similar in all sensitivity analyses, but heterogeneity $\left(\mathrm{I}^{2}\right)$ was decreased to $30 \%$ when only studies with a lower risk of bias (PEDro score $\geq 7$ ) were included (table 2 ). The pooled estimate of the effect of exercise on falls in residential care settings was more variable in the sensitivity analysis, reflecting greater uncertainty about the effectiveness of exercise on falls in this population (table 2).

The pooled effect of exercise was 0.47 in people with Parkinson's disease (95\% CI 0.30 to $0.73, \mathrm{p}=0.001, \mathrm{I}^{2} 65 \%, 6$ comparisons); 0.74 in people after stroke ( $95 \%$ CI 0.42 to 1.32 , $\mathrm{p}=0.31, \mathrm{I}^{2} 39 \%, 3$ comparisons); 0.55 in people with cognitive impairment (95\% CI 0.37 to $0.83, \mathrm{p}=0.004, \mathrm{I}^{2} 21 \%, 3$ comparisons) and 1.16 in people recently discharged from hospital (95\% CI 0.88 to $1.52, \mathrm{p}=0.30, \mathrm{I}^{2} 47 \%, 3$ comparisons) (figure 4 ). These estimates were essentially unchanged when only trials with a lower risk of bias (PEDro score $\geq 7$ ) were included (table 2).

\section{Exploration of impact of trial characteristics on the effect of exercise on falls}

In community-dwelling older people, greater intervention effects were seen in trials that included exercise programmes that aimed to provide a high challenge to balance (the ratio of rate ratios (RRR) obtained from the meta-regression was 0.85 $(95 \%$ CI 0.73 to $0.995, \mathrm{p}=0.04,28 \%$ of heterogeneity explained)) and 3 or more hours per week of prescribed exercise over the programme period (RRR $0.77,95 \%$ CI 0.65 to 0.91 , $\mathrm{p}=0.003, \quad 61 \%$ of heterogeneity explained; table 3 ). Multivariable meta-regression analysis found these factors to be independently associated with greater fall prevention effects and when combined, explained $76 \%$ of between-study heterogeneity (table 4). The modelled effect on falls of exercise programmes with neither of these variables was 0.90 (95\% CI 0.82 to 0.99 , $\mathrm{p}=0.03)$ and with both of these variables was $0.61(95 \%$ CI 0.53 to $0.72, \mathrm{p}<0.001$ ).

\section{Exploration of small study effects}

Visual inspection of the funnel plots (figure 5) suggested some asymmetry and thus a possibility of small study effects in the analyses of studies undertaken in community-dwellers, in residential care and in people with Parkinson's disease. We considered there to be too few studies in other clinical groups to enable exploration of small study effects. Egger's test found evidence of small study effects in community-dwellers $(p=0.02)$ but not the other analyses (residential care $p=0.09$, Parkinson's disease $\mathrm{p}=0.19$ ). These last two analyses may be underpowered, given the small numbers of studies involved.

\section{DISCUSSION}

This systematic review with meta-analysis provides strong evidence that exercise as a single intervention prevents falls in older people living in the community. The meta-regression suggests programmes that involve a high challenge to balance and include more than 3 hours/week of exercise have greater fall prevention 
Table 3 Results of meta-regression exploring the impact of trial-level characteristics on the effect of exercise on falls in general community-dwelling older populations

\begin{tabular}{|c|c|}
\hline $\begin{array}{l}\text { Variable tested in meta-regression analyses } \\
\text { (number of trials with this characteristic for dichotomous variables) }\end{array}$ & Coefficient $(95 \% \mathrm{Cl}), \mathrm{p}, \%$ hete \\
\hline \multicolumn{2}{|l|}{ Study design } \\
\hline PEDro score*, number/10 & $1.01(0.93$ to 1.08$), 0.88,-5 \%$ \\
\hline Participants randomised, number & $1.00(1.00$ to 1.00$), 0.37,-2 \%$ \\
\hline Weeks of follow-up, number & $1.00(1.00$ to 1.00$), 0.07,18 \%$ \\
\hline \multicolumn{2}{|l|}{ Sample characteristic } \\
\hline Average age $>75$ years $(33)$ & $0.95(0.80$ to 1.12$), 0.52,-3 \%$ \\
\hline Control group fall rate, available for 65 comparisons & $0.99(0.90$ to 1.07$), 0.73,-9 \%$ \\
\hline Unselected population (37) & $1.02(0.87$ to 1.21$), 0.79,-7 \%$ \\
\hline \multicolumn{2}{|l|}{ Programme characteristic } \\
\hline Inclusion of moderate or high-intensity strength trainingt (28) & $0.97(0.82$ to 1.15$), 0.73,-4 \%$ \\
\hline Inclusion of high-intensity strength training (10) & 1.23 (0.96 to 1.57$), 0.11,9 \%$ \\
\hline Inclusion of moderate or high challenge balance training (47) & $0.85(0.71$ to 1.00$), 0.06,19 \%$ \\
\hline Inclusion of high challenge balance training (31) & $0.85(0.73$ to 1.00$), 0.04,28 \%$ \\
\hline Inclusion of walking training or practice (29) & $1.01(0.86$ to 1.20$), 0.87,-6 \%$ \\
\hline $2+$ hours per week of exercise $(40)$ & $0.98(0.83$ to 1.16$), 0.83,-6 \%$ \\
\hline $3+$ hours per week of exercise (20) & $0.77(0.65$ to 0.91$), 0.003,61 \%$ \\
\hline Better adherence to exercise§ (52) & 0.95 (0.79 to 1.13$), 0.54,-1 \%$ \\
\hline
\end{tabular}

*Coded using PEDro rating scale 14

tModerate intensity (40-60\% of the 1-repetition maximum (RM) that is, a weight so heavy that it can only be lifted once) or high intensity ( $>60 \% 1 \mathrm{RM}$ ).

¥Moderately challenging=two of the following criteria or highly challenging=all three criteria: movement of the centre of mass, narrowing of the base of support and minimising upper limb support.

§Greater than or equal to $75 \%$ participants attended $50 \%$ or more sessions and/or $>50 \%$ attendance rate.

Note: a meta-regression coefficient $<1$ indicates a greater impact of exercise on falls in trials with that characteristic; a negative number for percentage of heterogeneity explained reflects no heterogeneity explained; number of trials with a particular characteristic indicated for dichotomous meta-regression variables only; statistically significant comparisons shown in italics.

Table 4 Results of multivariable meta-regression exploring the impact of trial-level characteristics on the effect of exercise on falls in general community-dwelling older populations

\begin{tabular}{|c|c|c|}
\hline $\begin{array}{l}\text { Variables included in multivariable meta-regression } \\
\text { (number of trials with this characteristic) }\end{array}$ & $\begin{array}{l}\text { Effect on effect size, meta-regression coefficient } \\
(95 \% \mathrm{CI}), \mathrm{p} \text { value }\end{array}$ & $\begin{array}{l}\text { Effect on falls, IRR }(95 \% \mathrm{Cl}) \text {, } \\
\mathrm{p} \text { value }\end{array}$ \\
\hline Inclusion of high challenge balance training * (31) & $0.87(0.76$ to 1.00$), 0.04$ & $0.79(0.71$ to 0.88$),<0.001$ \\
\hline $3+$ hours per week of intervention (20) & $0.78(0.66$ to 0.92$), 0.004$ & 0.70 (0.60 to 0.83$),<0.001$ \\
\hline $\begin{array}{l}\text { Neither high challenge balance training or } 3+\text { hours per week } \\
\text { of intervention }\end{array}$ & & 0.90 (0.82 to 0.99), 0.03 \\
\hline High challenge balance training and 3+ hours per week of intervention & & $0.61(0.53$ to 0.72$),<0.001$ \\
\hline
\end{tabular}

effects. The pooling of results from 62 trials across a range of countries suggests the results can be broadly generalised to community-dwelling older people, although it is acknowledged that few studies were undertaken in low-income and middle-income countries. Fewer studies have been undertaken in residential care settings and in people with particular clinical conditions, so there is less certainty about the impact of exercise as a single intervention in these groups. There is currently no evidence that exercise as a single intervention can prevent falls in residential care settings, among stroke survivors or among people recently discharged from hospital. There was evidence of a fall prevention effect in community-dwelling people with Parkinson's disease and people with cognitive impairment, but this needs to be confirmed with further studies. Our updated recommendations for fall prevention practice are shown in box 1 .

In previous versions ${ }^{8} 9$ of this systematic review with meta-analysis, we pooled trials from community and residential care settings and people with different health conditions to provide sufficient trials to enable meta-regression to be undertaken to explore characteristics of more effective interventions.
The number of trials has doubled since our previous update, so this is no longer necessary. The large number of trials now enables separate analysis by setting and condition. Given the likely heterogeneity between people living in different settings and with different health conditions, separate analysis by setting is clinically justifiable. Similarly, we previously combined studies that reported the effect of exercise on the proportion of fallers in intervention versus control groups with studies that reported effects on number of falls. However, as interventions may have different impacts on the proportion of fallers and the number of falls this approach is not ideal and is no longer necessary due to the additional studies now available for analysis.

Two-thirds (76\%) of the between-trial heterogeneity $\left(\mathrm{I}^{2} 47 \%\right)$ in the community-dwelling older people could be explained by the presence of two exercise programme characteristics: a high challenge to balance and more than 3 hours/week of exercise. Exercise programmes that contained these components reduced the rate of falls by $39 \%$. This is consistent with the results of our previous review, ${ }^{9}$ but in the current review, a higher dose of exercise and higher challenge to balance differentiated more 


\section{A}

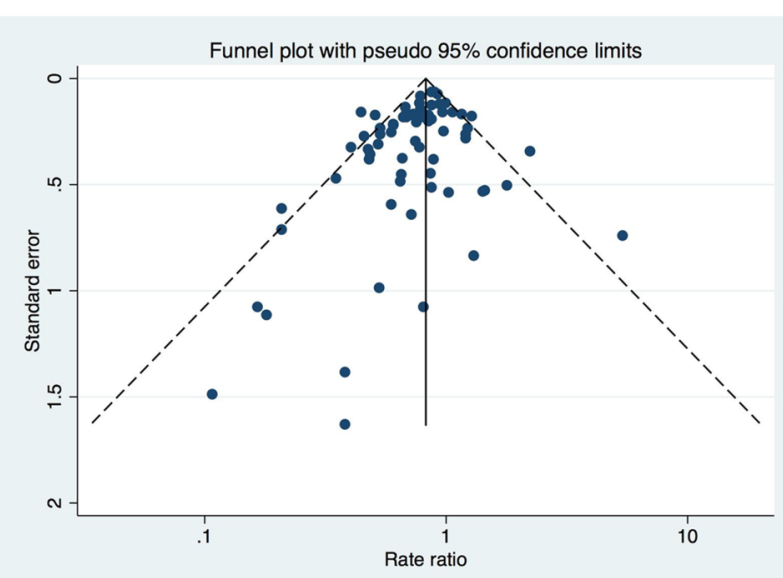

B

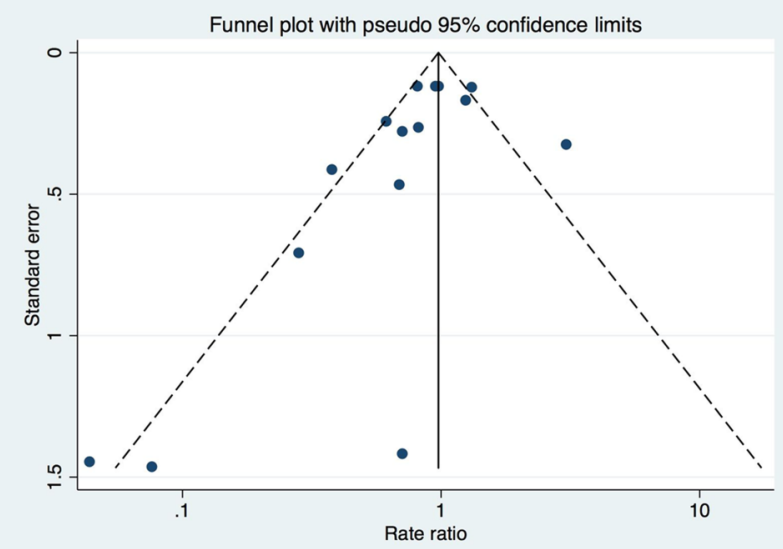

C

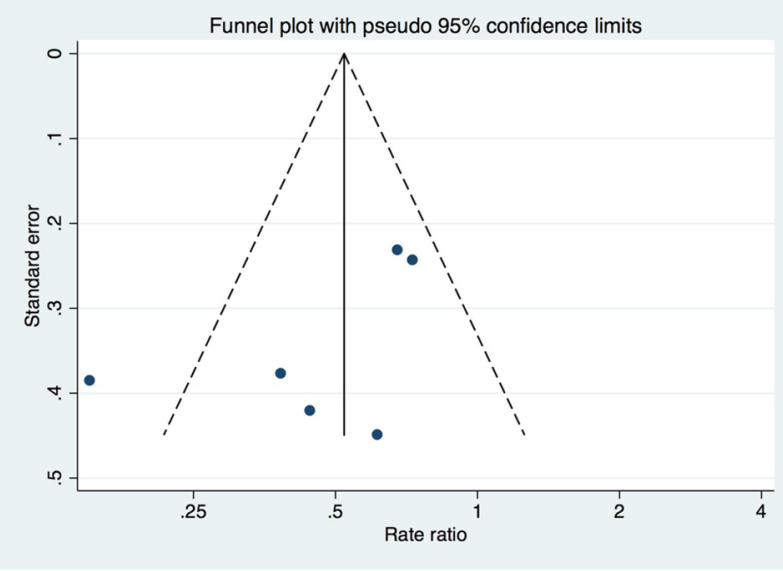

Figure 5 Funnel plots showing SE and effect size (log rate ratio) in trials of exercise for fall prevention undertaken among (A) general community dwellers, (B) residential care residents and (C) community dwellers with Parkinson's disease.

effective and less effective trials. This information can be used in the design of future fall prevention programmes. A safe challenge to balance can be delivered in diverse ways by home exercise, group exercise and Tai Chi. ${ }^{7}$ In situations with resource constraints, group exercise programmes may need to be supplemented with home exercise to achieve a higher dose of overall exercise. It is important to note that the exercise programmes tested in the included trials were mostly individually prescribed by trained health or exercise professionals to minimise the risk of harm (including falls) while exercising. Indeed when implementing fall prevention interventions, one should consider
Box 1 Updated recommendations for fall prevention practice in community-dwelling older people

1. Exercise programmes should aim to provide a high challenge to balance. Choose exercises that involve safely:

A. reducing the base of support (eg, standing with two legs close together, standing with one foot directly in front of the other, standing on one leg);

B. moving the centre of gravity and controlling body position while standing (eg, reaching, transferring body weight from one leg to another, stepping up onto a higher surface); and

C. standing without using the arms for support, or if this is not possible then aim to reduce reliance on the upper limbs (eg, hold onto a surface with one hand rather than two, or one finger instead of the whole hand)

2. At least 3 hours of exercise should be undertaken each week

3. Ongoing participation in exercise is necessary or benefits will be lost

4. Fall prevention exercise should be targeted at the general community as well as community-dwellers with an increased risk of falls

5. Fall prevention exercise may be undertaken in a group or home-based setting

6. Walking training may be included in addition to balance training but high-risk individuals should not be prescribed brisk walking programmes

7. Strength training may be included in addition to balance training

8. Exercise providers should make referrals for other risk factors to be addressed

9. Exercise as a single intervention may prevent falls in people with Parkinson's disease or cognitive impairment. There is currently no evidence that exercise as a single intervention prevents falls in stroke survivors or people recently discharged from hospital. Exercise should be delivered to these groups by providers with particular expertise.

characteristics of successful interventions in addition to features that differentiate successful from less successful interventions.

Unlike the previous versions of this review, ${ }^{89}$ the presence of a walking programme was not associated with a reduction in intervention effectiveness. A recent trial ${ }^{20}$ evaluating the effectiveness of a walking programme supported by telephone coaching found that this programme did not prevent falls. Taken together with an earlier trial ${ }^{21}$ in which the risk of falls was increased from a brisk walking programme, and the meta-regression findings, we suggest walking should not be prescribed as a single fall prevention intervention and high-risk older people should not be told to walk briskly. However, given the popularity of walking, ${ }^{22}$ its other health benefits and the inclusion of individually prescribed walking in some successful interventions (such as the Otago Home Exercise Programme), ${ }^{23}$ we suggest that walking programmes may be carefully prescribed to older people in addition to other fall prevention exercises.

The lack of a fall prevention effect from exercise as a single intervention in residential care settings is consistent with the results of the Cochrane systematic review of fall prevention in aged care facilities. ${ }^{24}$ The more dependent population in aged care facilities may require additional interventions targeting multiple health conditions and impairments. This does not mean 
there is no role for exercise as a component of a fall prevention programme in residential care. Several individual trials that have found fall prevention effects in residential care settings have included exercise as a key component of the intervention, but have also included other strategies, including education of staff and attention to the environment. ${ }^{25}$ More work is required to establish the optimal fall prevention approach in residential care, but exercise as a single intervention cannot be recommended as a fall prevention strategy at present. There are other benefits of maintaining function in this population such as enhanced quality of life, decreased care needs and fewer complications such as chest infections and pressure injuries and appropriately designed exercise programmes may be able to achieve this.

There have been relatively few trials of fall prevention strategies in people with particular clinical conditions or fall risk factors. We found promising results for the prevention of falls with exercise as single intervention in people with cognitive impairment and Parkinson's disease, but more trials are required in these groups. While we did not find evidence of fall prevention effects of exercise as a single intervention in people recently discharged from hospital, there is evidence that home safety interventions can prevent falls in this groups. ${ }^{7}$ It is not yet known how to prevent falls in stroke survivors. ${ }^{26}$ As exercise has been found to have other benefits for these groups, including improved mobility, ${ }^{27} 28$ future trials could investigate the combination of exercise and other fall prevention interventions.

This systematic review has some limitations. In particular, the meta-regression should be interpreted with caution as the 'effects' estimated with meta-regression are based on nonrandomised comparisons between studies, so they are potentially confounded. ${ }^{12}$ Individual participant data meta-analysis may better enable investigation of the impact of sample characteristics such as age and fall risk status on intervention effectiveness. Coding of the exercise programme components was undertaken on the information available in the published papers and therefore, there is the potential for inaccurate interpretation. Confidence in the results of the meta-analysis in community-dwellers is increased by the stability of the estimates of effect when fixed-effects meta-analysis and sensitivity analyses were undertaken. Although the funnel plot asymmetry and Eggers' test suggest this analysis may be affected by small study effects, this was not supported by the results of the sensitivity analysis when the smaller studies were excluded or by the lack of a significant effect of sample size on effect size in the meta-regression. However, the risk of small study effects in the residential care settings and in community-dwellers with Parkinson's disease will require further exploration when more trials become available.

In conclusion, this updated review confirms that exercise as a single intervention can prevent falls in community-dwelling older people. Programmes that challenge balance and are of a higher dose have larger effects. The impact of exercise as a single intervention in other clinical groups and aged care facility residents requires further investigation. Promising results have been obtained in people with Parkinson's disease and cognitive impairment.

Twitter Follow Catherine Sherrington at @CathieSherr

Acknowledgements Claire Mok and Angie Baba provided valuable assistance with literature searching and data extraction, respectively.

Contributors CS, AT, JW, RGC, RDH, JCTC and SRL contributed to the design of this study. CS, ZAM, NF and SSP contributed to literature searching and eligible study identification. CS, ZAM, NF, SSP, JW, JCTC and SRL contributed to data extraction. CS and RDH undertook data analysis. CS, ZAM, NF, SSP, AT, JW, RGC,
RDH, JCTC and SRL contributed to manuscript drafting and/or editing and reviewed the final version.

Funding CS, AT, RDH and SRL receive salary funding from the Australian National Health and Medical Research Council.

Competing interests None declared.

Provenance and peer review Not commissioned; externally peer reviewed.

Data sharing statement The authors are willing to share data with receipt of a written request to the corresponding author

\section{REFERENCES}

1 Alamgir H, Muazzam S, Nasrullah M. Unintentional falls mortality among elderly in the United States: time for action. Injury 2012;43:2065-71.

2 Rubinstein LZ. Falls in older people: epidemiology, risk factors and strategies for prevention. Age Aging 2006;35:ii37-41.

3 Lord SR, Ward JA, Williams P, et al. An epidemiological study of falls in older community-dwelling women: the Randwick falls and fractures study. Aust J Public Health 1993;17:240-5.

4 Murray C, Vos T, Lozano R, et al. Disability-adjusted life years (DALYs) for 291 diseases and injuries in 21 regions, 1990-2010: a systematic analysis for the Global Burden of Disease Study 2010. Lancet 2012:380:2197-223.

5 National Institute for Health and Care Excellence. Falls in older people: assessing risk and prevention. 2013. http://www.nice.org.uk/guidance/cg161 (accessed 29 Oct 2015).

6 Australian Commission on Safety and Quality in Health Care. Safety and Quality Improvement Guide Standard 10: Preventing Falls and Harm from Falls. 2012. http://www.safetyandquality.gov.au/wp-content/uploads/2012/10/Standard10_Oct_ 2012 WEB.pdf (accessed 30 Oct 2015)

7 Gillespie LD, Robertson MC, Gillespie WJ, et al. Interventions for preventing falls in older people living in the community. Cochrane Database Syst Rev 2012(9): CD007146.

8 Sherrington C, Whitney JC, Lord SR, et al. Effective exercise for the prevention of falls: a systematic review and meta-analysis. J Am Geriatr Soc 2008;56:2234-43.

9 Sherrington C, Tiedemann A, Fairhall N, et al. Exercise to prevent falls in older adults: an updated meta-analysis and best practice recommendations. N S W Public Health Bull 2011;22:78-83.

10 Campbell AJ, Robertson MC. Rethinking individual and community fall prevention strategies: a meta-regression comparing single and multifactorial interventions. Age Ageing 2007;36:656-62.

11 Davis JC, Robertson MC, Ashe MC, et al. Does a home-based strength and balance programme in people aged $>$ or $=80$ years provide the best value for money to prevent falls? A systematic review of economic evaluations of falls prevention interventions. Br J Sports Med 2010:44:80-9.

12 Thompson SG, Higgins JP. How should meta-regression analyses be undertaken and interpreted? Stat Med 2002;21:1559-73.

13 Liberati A, Altman DG, Tetzlaff J, et al. The PRISMA statement for reporting systematic reviews and meta-analyses of studies that evaluate health care interventions: explanation and elaboration. PLoS Med 2009:6:e1000100.

14 Maher CG, Sherrington C, Herbert RD, et al. Reliability of the PEDro scale for rating quality of randomized controlled trials. Phys Ther 2003;83:713-21.

15 Physiotherapy Evidence Database. PEDro. http://www.pedro.org.au (accessed 30 Oct 2015).

16 Harris RJ, Bradburn MJ, Deeks JJ, et al. Metan: fixed- and random-effects meta-analysis. Stata J 2008:8:3-28

17 Harbord RM, Higgins JP. Meta-regression in Stata. Stata J 2008:8:493-1519.

18 Lord SR, Castell S, Corcoran J, et al. The effect of group exercise on physical functioning and falls in frail older people living in retirement villages: a randomized, controlled trial. J Am Geriatr Soc 2003;51:1685-92.

19 Morgan RO, Virnig BA, Duque $M$, et al. Low-intensity exercise and reduction of the risk for falls among at-risk elders. J Gerontol A Biol Sci Med Sci 2004;59:1062-7.

20 Voukelatos A, Merom D, Sherrington C, et al. The impact of a home-based walking program on falls in older people: the Easy Steps randomised controlled trial. Age Ageing 2015;44:377-83

21 Ebrahim S, Thompson PW, Baskaran V, et al. Randomized placebo-controlled trial of brisk walking in the prevention of postmenopausal osteoporosis. Age Ageing 1997:26:253-60.

22 Merom D, Pye $\mathrm{V}$, Macniven $\mathrm{R}$, et al. Prevalence and correlates of participation in fall prevention exercise/physical activity by older adults. Prev Med 2012;55: 613-17.

23 Robertson MC, Campbell AJ, Gardner MM, et al. Preventing injuries in older people by preventing falls: a meta-analysis of individual-level data. J Am Geriatr Soc 2002:50:905-11.

24 Cameron ID, Murray GR, Gillespie LD, et al. Interventions for preventing falls in older people in care facilities and hospitals. Cochrane Database Syst Rev 2012;(1):CD005465.

25 Becker $\mathrm{C}$, Kron M, Lindemann U, et al. Effectiveness of a multifaceted intervention on falls in nursing home residents. J Am Geriatr Soc 2003;51:306-13. 
26 Batchelor $\mathrm{F}$, Hill K, Mackintosh $\mathrm{S}$, et al. What works in falls prevention after stroke?: a systematic review and meta-analysis. Stroke 2010;41:1715-22.

27 Dean CM, Rissel C, Sherrington C, et al. Exercise to enhance mobility and prevent falls after stroke: the community stroke club randomised trial. Neurorehabil Neural Repair 2012;36:1046-57.
28 Sherrington C, Lord SR, Vogler CM, et al. A post-hospital home exercise program improved mobility but increased falls in older people: a randomised controlled trial. PLOS ONE 2014;9:e104412.

$\underline{\square}$

$c$

$\frac{c}{8}$

3

?.

$\stackrel{\vec{P}}{\stackrel{\vec{p}}{9}}$

듬

$\frac{\bar{\sigma}}{\bar{\sigma}}$

ळ

$\vec{\circ}$

$\overrightarrow{\vec{\omega}}$ 